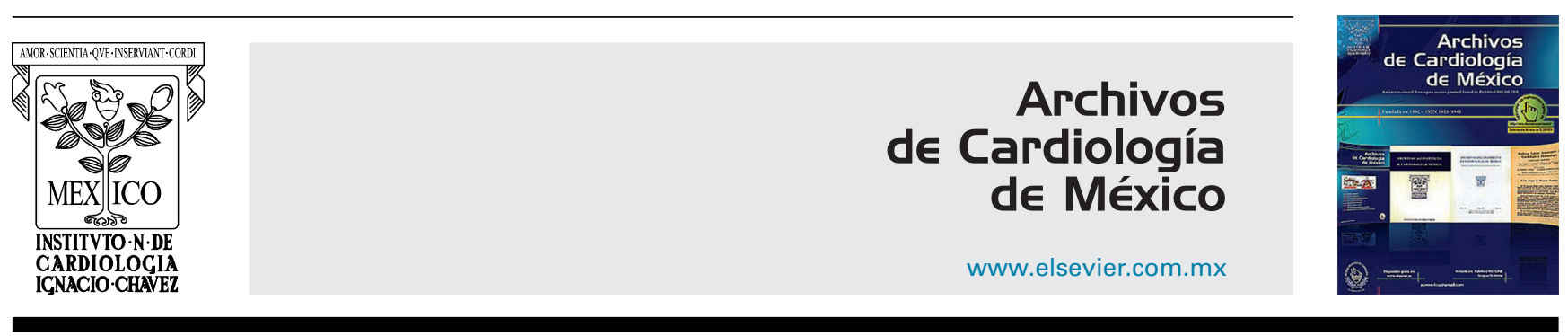

IMAGEN EN CARDIOLOGÍA

\title{
Miocardio no compacto presentándose clínicamente con insuficiencia cardíaca
}

\section{Not compacted myocardium debuting clinically with heart failure}

\author{
Martha Abigaíl Reyes Villatoro*
}

Departamento de Electrofisiología, Instituto Nacional de Cardiología Ignacio Chávez, Facultad de Medicina, Universidad Nacional Autónoma de México, México D.F., México

Recibido el 31 de octubre de 2015; aceptado el 7 de abril de 2016

Miocardio ventricular no compacto es una miocardiopatía congénita que es causada por el cese de compactación en la embriogénesis temprana del endocardio y el miocardio.

Ya que en el desarrollo embrionario inicial, el miocardio es una red flexible de fibras entrelazadas separadas por profundos huecos que enlazan el miocardio con la cavidad ventricular izquierda. Gradual «compactación» de esta malla esponjosa de fibras y recesos intertrabeculares, 0 «sinusoides», se produce entre las semanas $5 . \underline{a}$ y $8 . \underline{a}$ de la vida embrionaria, que procede del epicardio a endocardio y desde la base del corazón al ápex. Pertenece a las miocardiopatías no clasificables. Esta anormalidad, a menudo se asocia con otros defectos cardíacos congénitos ${ }^{1-3}$.

El ventrículo izquierdo es principalmente afectado, pero se han reportado casos con afección biventricular; el ventrículo derecho se ve afectado en menos de la mitad de los casos.

\footnotetext{
* Autor para correspondencia. Calle Juan Badiano 1, Tlalpan, Belisario Domínguez, sección XVI, Código postal 14080, Ciudad de México, D.F., México, Teléfono: 5655-2924, Fax: +5573 0994.

Correo electrónico: martyreyes@hotmail.com
}

El diagnóstico correcto es a menudo retrasado debido a la falta de conocimiento acerca de esta infrecuente enfermedad y su similitud con otras enfermedades del miocardio y endocardio. Muchos casos de ventrículo izquierdo no compacto se han confundido inicialmente con diagnósticos de miocardiopatía hipertrófica, dilatada o miocardiopatía restrictiva (fibrosis endomiocárdica).

Su presentación clínica con insuficiencia cardíaca se ha reportado entre el 30-73\%, con frecuencia pasa desapercibida y usualmente solo se detecta en centros médicos con experiencia en el diagnóstico y tratamiento de miocardiopatías ${ }^{4}$.

El diagnóstico por ecocardiograma se realiza por una relación en fin de sístole entre miocardio no compactado y compactado $>2$.

En resonancia magnética cardíaca el criterio más aceptado utiliza el cociente mayor de 2.3 entre miocardio no compacto/compacto, con una sensibilidad diagnóstica del $86 \%$ y una especificidad del $99 \%^{5-7}$.

La terapia debe ir dirigida a las complicaciones que presente cada paciente: insuficiencia cardíaca, arritmias y eventos embólicos. Los pacientes con fallo cardíaco pueden ser tratados con las guías de manejo convencionales. 


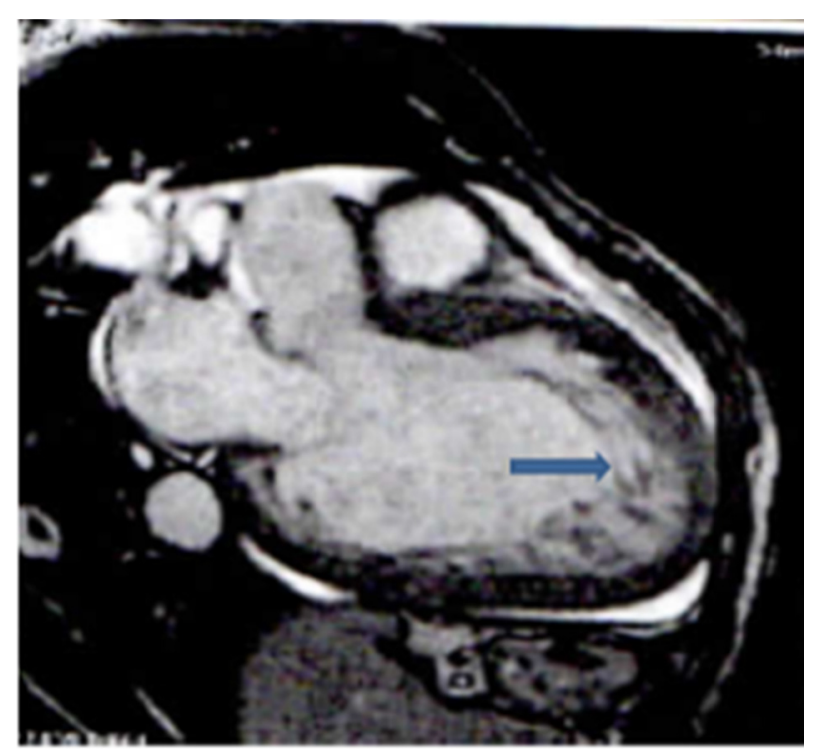

Figura 1 Resonancia magnética cardíaca. Eje largo de 3 cámaras que muestra la imagen de doble capa reflejando miocardio no compacto/compacto.

En insuficiencia cardíaca refractaria a tratamiento debe ser considerado el trasplante cardíaco. La terapia de resincronización cardíaca ha mostrado remodelación inversa del MNC.

El desfibrilador implantable ha sido utilizado en la prevención primaria y secundaria de arritmias letales, ya que estos pacientes tienen alta probabilidad de muerte súbita (clase IIb AHA/ACC para prevención primaria).

Varón de 65 años, sin antecedentes cardiovasculares contributorios, con 4 meses de edema ascendente de extremidades inferiores, 2 meses de edema facial, disnea de moderados a mínimos esfuerzos, palpitaciones esporádicas y tos no productiva. Al examen físico con presión arterial de $170 / 90 \mathrm{mmHg}$, frecuencia cardíaca $78 \mathrm{lpm}$, ingurgitación yugular grado । y escasos crépitos en ambas bases pulmonares. Ruidos cardíacos arrítmicos por latidos ectópicos con pausa compensatoria, primer ruido único, tercer ruido intermitente. Soplo regurgitante protosistólico grado ॥/ Iv en foco mitral.

Electrocardiograma en ritmo sinusal, con bloqueo completo de rama izquierda del haz de His. La serología para Chagas fue negativa.

Ecocardiograma compatible con ventrículo izquierdo no compacto, con severa disfunción sistólica e insuficiencia mitral leve.

La resonancia magnética a través de las secuencias eco de gradiente no observó alteración de la contractilidad segmentaria, la hipocinesia reportada es generalizada, con imagen de doble capa (fig. 1), con una relación miocardio no compacto/compacto de 2.4, medido en diástole en pared anterior, en eje corto segmento basal, medio y apical (fig. 2). Con positividad para miocardio no compacto $^{5-7}$.

Ventrículo izquierdo dilatado, con índice de volumen telediastólico de $283 \mathrm{ml} / \mathrm{m}^{2}$ e índice de volumen telesistólico de $235 \mathrm{ml} / \mathrm{m}^{2}$, FEVI del $17 \%$.

Ventrículo derecho de morfología normal con índice de volumen telediastólico de $102 \mathrm{ml} / \mathrm{m}^{2}$ e índice de volumen telesistólico de $50 \mathrm{ml} / \mathrm{m}^{2}$.

El estudio de viabilidad miocárdica con técnica de T1, eco de gradiente, inversión-recuperación posterior a la administración de gadolinio, sin presencia de fibrosis a nivel miocárdico.

Paciente con evolución clínica favorable, tratado con enalapril, carvedilol, digoxina, espironolactona, furosemida y aspirina.

Por su potencial arritmogénico, fue valorado por el servicio de electrofisiología y se indicó implante de desfibrilador automático como prevención primaria de muerte súbita más resincronización cardíaca.

Se reporta un caso de miocardio no compacto con afección del ventrículo izquierdo, de forma esporádica, comenzando clínicamente con insuficiencia cardíaca, que no se asocia a otras anomalías congénitas, cuya incidencia local es del $18 \%$, menor a la reportada en otros estudios ${ }^{4}$.

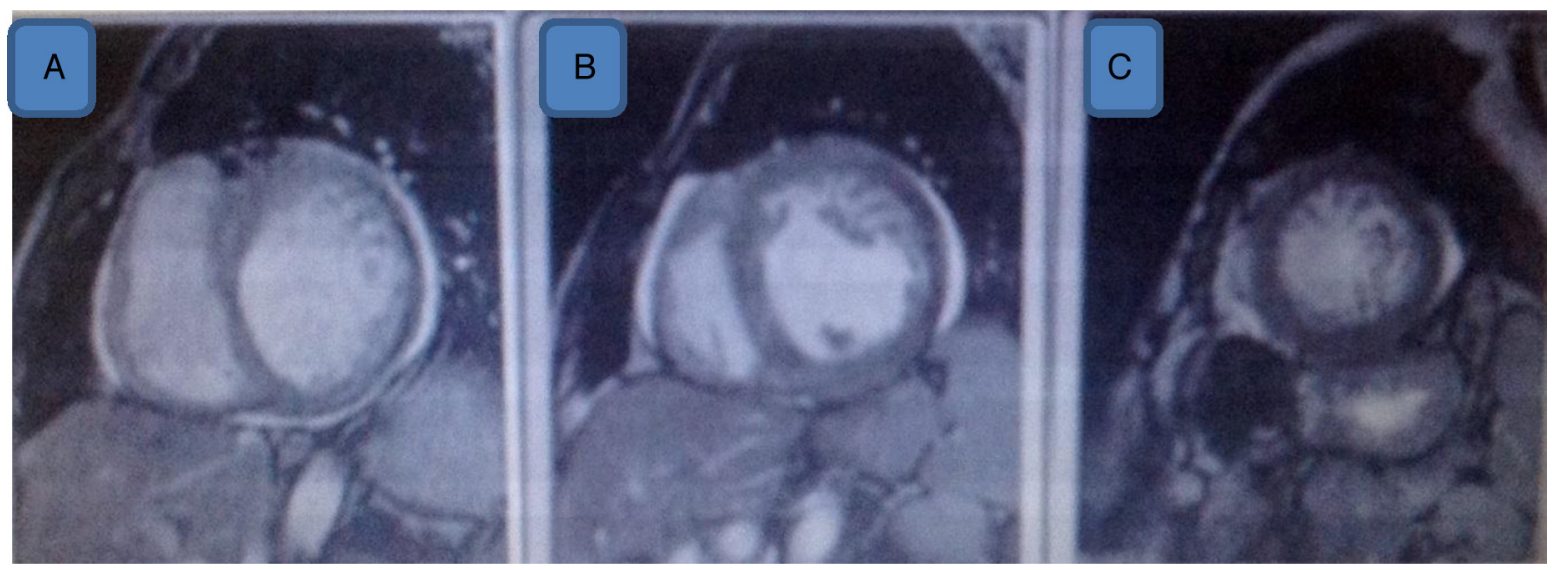

Figura 2 Resonancia magnética cardíaca. Diagnóstico de miocardio no compacto por relación miocardio no compacto/compacto de 2.4 medido en eje corto a nivel basal (A), medio (B) y apical (C) 


\section{Responsabilidades éticas}

Protección de personas y animales. Los autores declaran que para esta investigación no se han realizado experimentos en seres humanos ni en animales.

Confidencialidad de los datos. Los autores declaran que en este artículo no aparecen datos de pacientes.

Derecho a la privacidad y consentimiento informado. Los autores declaran que en este artículo no aparecen datos de pacientes.

\section{Financiación}

No se recibió patrocinio de ningún tipo para llevar a cabo este artículo.

\section{Conflicto de intereses}

El autor declara no tener ningún conflicto de intereses.

\section{Bibliografía}

1. Restrepo G, Castaño R, Mármol A. Ventrículo izquierdo no compacto/ventrículo izquierdo hipertrabeculado. Rev Col Cardiol. 2005; 12:20-4.

2. Elias J, Valadão W, Kuniyoshi R, et al. Isolated noncompaction of the myocardium. Arq Bras Cardiol. 2000;74:253-61.

3. Stöllberger C, Finsterer J. Left ventricular hypertrabeculation/noncompaction. J Am Soc Echocardiogr. 2004;17:91-100.

4. Brian C, Weiford MD, Vijay D, et al. Noncompaction of the ventricular myocardium. Circulation. 2004;109:2965-71.

5. Oechslin EN, Attenhofer Jost CH, Rojas JR, et al. Long-term follow-up of 34 adults with isolated left ventricular noncompaction: A distinct cardiomyopathy with poor prognosis. J Am Coll Cardiol. 2000;36:493-500.

6. Jenni R, Wyss CA, Oechslin EN, et al. Isolated ventricular noncompaction is associated with coronary microcirculatory dysfunction. J Am Coll Cardiol. 2002;39:450-4.

7. Petersen SE, Selvanayagam JB, Wiesmann F, et al. Left ventricular non-compaction: Insights from cardiovascular magnetic resonance imaging. J Am Coll Cardiol. 2005;46:101-5. 